\title{
COFINALITY IN NORMAL ALMOST COMPACT SPACES
}

\author{
W. FLEISSNER, J. KULESZA, AND R. LEVY
}

(Communicated by R. Daniel Mauldin)

\begin{abstract}
A regular space is said to be a NAC space if, given any pair of disjoint closed subsets, one of them is compact. The standard example of a noncompact NAC space is an ordinal space of uncountable cofinality. The cofinality of an arbitrary noncompact NAC space is defined, and the extent to which cofinality in NAC spaces behaves like cofinality of ordinal spaces is discussed.
\end{abstract}

The notion of cofinality is fundamental in the study of ordered topological spaces. Our goal is to investigate the notion of cofinality in topological spaces. Without a linear order, we lose our sense of direction, so we study spaces where "we can approach infinity from only one direction." Even with a linear order, we must be somewhat careful; after all, every infinite discrete space has a linear order that has a cofinality, but different linear orders on a given discrete space can give different cofinalities. We want to look at analogues not of arbitrary ordered spaces, but rather those in which the cofinality can be described in terms of the topology. This idea is made precise by looking at spaces having a unique free closed ultrafilter, spaces that we call NAC spaces. We see that the theory develops smoothly for spaces that have the additional property that small subsets have compact closure, but the general theory has more questions than answers.

In $\S 1$ we give some preliminary definitions. Section 2 introduces two notions analogous to the cofinality of a linear ordered set. Several examples are given and discussed in $\S 3$. In $\S 4$ we discuss normality of products and continuous images of normal almost compact spaces. Section 5 gives other results.

\section{Preliminary definitions}

All spaces we deal with are assumed to be at least regular. A Tychonov space $X$ is almost compact if $\beta X \backslash X$ has at most one element. If $X$ is almost compact but not compact, the unique element of $\beta X \backslash X$ is denoted by $\infty_{X}$, or if no ambiguity can result, simply $\infty$. Examples are the space $\omega_{1}$ of countable

Received by the editors September 15, 1989.

1980 Mathematics Subject Classificaiion (1985 Revision). Primary 54D30.

Key words and phrases. NAC space, cofinality. 
ordinals, and the Tychonov plank $T$. The former is normal and the latter is not. A regular space is normal and almost compact if and only if at least one of any pair of disjoint closed sets is compact. We call a noncompact, normal, almost compact space a NAC space. A NAC space is locally compact and countably compact. Every noncompact closed subset of a NAC space is NAC. Furthermore, if $X$ is NAC and $f: X \rightarrow Y$ is a continuous surjection, then either $Y$ is compact or $Y$ is NAC and $f$ is a perfect mapping. (Recall that a map $f: X \rightarrow Y$ is perfect if $f$ is closed mapping such that the preimage of each point of $Y$ is compact.) For Tychonov spaces, $X$ and $Y, f: X \rightarrow Y$ is perfect if and only if $\left.\beta f^{\rightarrow}(\beta X \backslash X)=\beta Y \backslash Y\right)$.

If $X$ is a topological space a closed filterbase on $X$ is a collection $\mathscr{F}$ of closed subsets of $X$ having the finite intersection property. A closed filter on $X$ is a closed filterbase that contains any closed superset of any of its elements. The notions of $z$-filterbase and $z$-filter are defined analogously with zero sets repiacing closed sets in the definitions. A closed filterbase or $z$-filterbase is free if it has empty intersection. A filterbase, or closed filterbase, or $z$-filterbase is well ordered if it has the form $\left\{F_{\alpha}: \alpha<\kappa\right\}$ where $F_{\gamma} \supseteq F_{\alpha}$ if $\gamma<\alpha$.

We use the standard (see for example [E]) notions of character, $\chi(p, X)$ and $\chi(X)$, pseudocharacter, $\psi(p, X)$ and $\psi(X)$, and tightness, $\tau(p, X)$ and $\tau(X)$. Because tightness is a sup that may not be attained, we sometimes instead use $\tau^{+}(X)$, defined to be the least $\kappa$ such that whenever $p \in X, A \subseteq X$, and $p \in \mathrm{Cl}_{X} A$, there is a subset of $A$ having cardinality less than $\kappa$ and whose closure contains $p$. If $\kappa$ is a cardinal, a space $X$ is $\kappa$-bounded [respectively, $<\kappa$-bounded] if every subset of $X$ having cardinality at most [respectively, fewer than] $\kappa$ elements has compact closure in $X$.

\section{CofinAlity AND CHARACTER AT $\infty$}

Suppose that $X$ is a NAC space. Define $\operatorname{cf}(X)$ to be the least cardinal of a free closed filterbase on $X$. We will call $\mathrm{cf}(X)$ the cofinality of $X$. Note that $X$ always has a well-ordered free closed filterbase of cardinality $\operatorname{cf}(X)$, because given an arbitrary closed free filterbase $\mathscr{F}=\left\{F_{\alpha}: \alpha<\operatorname{cf}(X)\right\}$, we may replace $F_{\alpha}$ by $\cap\left\{F_{\lambda}: \lambda<\alpha\right\}$ for each $\alpha$. It follows easily that the cofinality of any NAC space is a regular cardinal. Define $\operatorname{zf}(X)$ to be the least cardinal of a free $z$-filterbase on $X$. Since a NAC space is countably compact, and since it is easier for sets to be closed than to be zero sets, for an arbitrary NAC space $X$, we have $\omega_{1} \leq \operatorname{cf}(X) \leq \operatorname{zf}(X)$. Notice that if $\kappa$ is an ordinal of uncountable cofinality, then the cofinality of $\kappa$ is the same as both $\operatorname{cf}(\kappa)$ and $\operatorname{zf}(\kappa)$.

Proposition 2.1. Let $X$ be a NAC space. Then the following cardinals are equal: $\mathrm{zf}(X), \chi(\infty, \beta X), \psi(\infty, \beta X)$, and the least cardinal of a family $\mathscr{K}$ of compact subsets of $X$ with $\bigcup \mathscr{K}=X$.

Proof. The equality $\operatorname{zf}(X)=\chi(\infty, \beta X)=\psi(\infty, \beta X)$ follows from the fact that zero-sets are $G_{\delta}$ sets and that in compact spaces, the pseudocharacter and 
character coincide. Observe that a subset $K$ of $X$ is compact if and only if $\beta X \backslash K$ is open in $\beta X$.

The simple proof of the following proposition is omitted.

Proposition 2.2. Suppose that $X$ is a NAC space. Let $\mathscr{F}$ be the family of noncompact closed subsets of $X . \mathscr{F}$ is a closed filterbase and any intersection of fewer than $\operatorname{cf}(X)$ elements of $\mathscr{F}$ is again an element of $\mathscr{F}$.

We now present a basic construction. Let $X$ be a NAC space, and let $\operatorname{cf}(X)=$ $\kappa$. Using $\mathscr{F}=\left\{F_{\alpha}: \alpha<\kappa\right\}$ a well-ordered free closed filterbase, we construct a $\kappa$-sequence in $X$. Let $p_{0}$ be an arbitrary element of $X$ and let $\gamma(0)=0$. For $\zeta<\kappa$, we suppose that points $p_{\alpha}$ of $X$ and ordinals $\gamma(\alpha)<\kappa$ are defined for all $\alpha<\zeta$. Let $K_{\zeta}=\mathrm{Cl}_{X}\left\{p_{\alpha}: \alpha<\zeta\right\}$. If $K_{\zeta}$ is compact, choose $\gamma(\zeta)$ so that $K_{\zeta} \cap F_{\gamma(\zeta)}=\varnothing$. Otherwise, let $\gamma(\zeta)=\sup \{\gamma(\alpha)+1: \alpha<\zeta\}$. Now choose $p_{\zeta} \in F_{\gamma(\zeta)}$. Then $\left\{p_{\zeta}: \zeta<\kappa\right\}$ is the desired sequence. We note that if $X$ is $<\kappa$-bounded, then the sequence $\left\{p_{\zeta}: \zeta<\kappa\right\}$ is a free sequence.

Proposition 2.3. Let $X, \kappa, p_{\zeta}$, and $\mathscr{F}$ be as in the preceding paragraph.

(a) Let $H_{\alpha}=\mathrm{Cl}_{X}\left\{p_{\zeta}: \alpha \leq \zeta<\kappa\right\}$. Then $\mathscr{H}=\left\{H_{\alpha}: \alpha<\kappa\right\}$ is a well-ordered free closed filterbase.

(b) If $q \in H_{0}$, then there is $B \subseteq \alpha<\kappa$ so that $\mathrm{Cl}_{X}\left\{P_{\zeta}: \zeta \in B\right\}$ is compact and contains $q$. If $X$ is $<\kappa$-bounded, we may choose $B=\alpha$.

(c) If $A \in[\kappa]^{\kappa}$ then $F(A)=\mathrm{Cl}_{X}\left\{p_{\zeta}: \zeta \in A\right\}$ is not compact.

Proof. Part (a) is immediate from the construction.

For (b) we note that since $\mathscr{H}$ is free, for $q \in H_{0}$ there is $\alpha<\kappa$ such that $q \notin H_{\alpha}$. Since $X$ is regular, there is an open set $U$ with $q \in U$ and $\mathrm{Cl}_{X} U \cap H_{\alpha}=\varnothing$. Set $B=\left\{\zeta \in \kappa: p_{\zeta} \in U\right\}$.

Statement (c) is obtained by observing that $\left\{\mathrm{Cl}_{X}\left\{p_{\zeta}: \zeta \in A \backslash \alpha: \alpha<\kappa\right\}\right.$ is a free closed filterbase.

Proposition 2.4. Let $X$ be a NAC space, $L$ a closed noncompact subset of $X$, and $f: X \rightarrow Y$ be a continuous mapping onto a noncompact space $Y$. Then $\operatorname{cf}(L)=\operatorname{cf}(X)=\operatorname{cf}(Y)$ and $\operatorname{zf}(L)=\operatorname{zf}(X)=\mathrm{zf}(Y)$.

Proof. The proofs of $\operatorname{cf}(L)=\operatorname{cf}(X)$ and $\operatorname{zf}(L) \leq \operatorname{zf}(X)$ are routine. The equality $\operatorname{zf}(Y)=\operatorname{zf}(X)$ follows from Proposition 2.1 because $f$ and $f^{\leftarrow}$ take compact sets to compact sets. $\operatorname{cf}(Y)=\operatorname{cf}(X)$ because $f$ and $f^{\leftarrow}$ take noncompact closed sets to noncompact closed sets.

Towards $\operatorname{zf}(X) \leq \operatorname{zf}(L)$, let $\left\{K_{\zeta}: \zeta<\operatorname{zf}(L)\right\}$ be a family of compact sets covering $L$. For $\zeta<\operatorname{zf}(L)$, find open subsets of $U_{\zeta}$ of $X$ and compact sets $H_{\zeta}$ so that $K_{\zeta} \subseteq H_{\zeta} \subseteq H_{\zeta}$. Set $F=\bigcap\left\{X \backslash U_{\zeta}: \zeta<\operatorname{zf}(L)\right\} . F$ is closed and disjoint from $L$, so $F$ is compact. Then $\{F\} \cup\left\{H_{\zeta}: \zeta<\operatorname{zf}(L)\right\}$ covers $X$.

Proposition 2.5. Let $X$ be a NAC space. Then $\operatorname{cf}(X) \leq \operatorname{zf}(X) \leq 2^{<\mathrm{cf}(X)}$. Thus, assuming $G C H, \operatorname{cf}(X)=\operatorname{zf}(X)$. If $X$ is $<\operatorname{cf}(X)$-bounded, then $\operatorname{cf}(X)=\operatorname{zf}(X)$ even if $G C H$ is not assumed. 
Proof. The inequality $\operatorname{cf}(X) \leq \operatorname{zf}(X)$ is immediate. Let $H_{0}$ be as in Proposition 2.3. Then $\mathrm{zf}(X)=\mathrm{zf}\left(H_{0}\right)$ by Proposition 2.4, and $\mathrm{zf}(H) \leq 2^{z \mathrm{cf}(X)}=$ $\left|[\kappa]^{<\kappa}\right|$ by Proposition 2.3(b). If $X$ is $<\kappa$-bounded, we can use $\kappa=\{\alpha: \alpha<$ $\kappa\}$ in place of $[\kappa]^{<\kappa}$.

\section{EXAMPLES}

In this section we discuss several classes of NAC spaces. Let $\kappa$ be an uncountable regular cardinal. NAC $(\kappa)=\{X: X$ is NAC and $\operatorname{cf}(X)=\kappa\}$. The spaces in $\Omega(\kappa)=\{X \in \mathrm{NAC}(\kappa): X$ is $<\kappa$-bounded $\}$ share many properties with the ordinals of cofinality $\kappa$, which are, of course, the canonical examples of NAC spaces. A still larger class if $\operatorname{FR}(\kappa)=\{X \in \mathrm{NAC}(\kappa)$ : There exists $L \in \Omega(\kappa)$ which is closed in $X\}$. By 2.5, if $X \in \Omega(\kappa)$, then $\operatorname{cf}(X)=\operatorname{zf}(X)$. Thus, by 2.4, if $X \in \mathrm{FR}(\kappa)$, then $\operatorname{cf}(X)=\operatorname{zf}(X)$.

Example 3.1. Eric van Douwen [vD1] constructed an interesting example of a NAC space $\Phi$ having no convergent sequence. The space $\Phi$ is defined to be $\beta\left(\left\{\lambda<\omega_{2}: \lambda\right.\right.$ has uncountable cofinality or is isolated $\left.\}\right) \backslash\left\{\omega_{2}\right\}$. Although the space $\Phi$ does not contain an ordinal space, it is a perfect preimage of an ordinal space. We remark that Fremlin [F] showed that assuming PFA perfect preimages of $\omega_{1}$ having small fibers contain copies of $\omega_{1}$ and in Remark 19 discusses a consistent example of a two-to-one perfect preimage of $\omega_{1}$ that does not contain a copy of $\omega_{1}$. See also the research announcement [BDFN].

Example 3.2. The closed long ray is a NAC space that is not a perfect preimage of an ordinal space, because it is connected. However, it is a perfect image of a perfect preimage of an ordinal space. More generally, one can show that any Dedekind complete linearly ordered topological space having a first element and uncountable cofinality is a perfect image of a perfect preimage of an ordinal of uncountable cofinality.

Example 3.3. In [FR] Franklin and Rajagopalan defined a countably compact space, which we denote by $\delta(N, \kappa)$, containing a dense countable discrete subset $N$ and such that $\delta(N, \kappa) \backslash N$ is (homeomorphic to) $\kappa$. (Without extra axioms of set theory, we can say only that $\kappa$ satisfies $\left.\omega_{1} \leq \kappa \leq \mathbf{c}\right)$. It is routine to check that $\delta(N, \kappa) \in \mathrm{FR}(\kappa) \backslash \Omega(\kappa)$.

Example 3.4. Assuming the axiom $\diamond$, Osteszewski [O] constructed a NAC space $\Theta$ that is hereditarily separable. Therefore no closed noncompact subset is $\omega$ bounded and $\tau(\beta \Theta)=\omega$.

Example 3.5. Nyikos (unpublished) showed that after iteratively adding $\omega_{1}$ dominating reals to a model $\mathscr{M}$ of set theory, there is a locally countable NAC space $Y$ of cardinality c that contains no perfect preimage of $\omega_{1}$. It follows that $\tau(\infty, \beta Y)=\omega<\operatorname{cf}(Y)=\omega_{1} \leq \mathbf{c}=\operatorname{zf}(Y)$. Because the cardinals and cardinal arithmetic of $\mathscr{M}$ are preserved, if $\mathrm{CH}$ fails in $\mathscr{M}$, then $\operatorname{cf}(Y)<\operatorname{zf}(Y)$ 
in the extension $\mathscr{M}[G]$, and if $\mathrm{c}$ is singular in $\mathscr{M}$ then $\operatorname{zf}(Y)$ is singular in $\mathscr{M}[G] . Y$ has well-ordered free closed filterbases of types $\omega_{1}$ and $\mathbf{c}$.

More examples are needed! Is every $X \in \Omega(\kappa)$ a perfect image of a perfect preimage of $\kappa$ ? Are there higher cardinal analogues of $\delta(N, \kappa)$ ? Specific versions of this question are: Is $\operatorname{FR}(\kappa) \backslash \Omega(\kappa)$ empty for $\kappa>c$ ? If a NAC space is $\omega$-bounded, is it $\alpha$-bounded for all $\alpha<\operatorname{cf}(X)$ ? If $X \in \mathrm{NAC}(\kappa)$ for $\kappa>\mathbf{c}$, does $\infty$ have a well-ordered base in $\beta X$ ?

We can also ask if there are higher cardinal and/or ZFC analogues of $\Theta$. Specific versions of this question are: Does $V=L$ imply that $\operatorname{NAC}(\kappa) \backslash \Omega(\kappa) \neq$ $\varnothing$ for all uncountable regular $\kappa$ ? Is it consistent with ZFC that $\tau(\infty, \beta X)=$ $\operatorname{cf}(X)$ for all NAC spaces $X$ ?

We recall the well-known question: Is it true in ZFC that $\omega^{*} \backslash\{p\}$ (or $\beta \omega \backslash\{p\}$ ) is nonnormal for every $p \in \omega^{*}$ ? A negative answer to this question would give a surprising NAC space. We do not know the answer to the following related questions: Does there exist a realcompact space $X$ and point $p \in X^{*}$ such that $\beta X \backslash\{p\}$ is normal, that is, such that $\beta X \backslash\{p\}$ is a NAC space? We note that the question is equivalent to the same question with $X$ assumed to be $\sigma$-comapct, or locally compact and $\sigma$-compact, or Lindelöf.

\section{NORMALITY OF PRODUCTS AND IMAGES}

In this section we discuss the relationship between cofinality in NAC spaces and normality of products and continuous images. We see that spaces in the class $\operatorname{FR}(\kappa)$ behave like $\kappa$ with respect to products. However, in the more general setting of $\operatorname{NAC}(\kappa)$, some of the product theorems do not hold, at least not consistently.

Proposition 4.1. Suppose that $X \in \mathrm{NAC}(\kappa)$.

(a) If $\mathscr{V}$ is an open cover of $X$ and $|\mathscr{V}|<\kappa$, then $\mathscr{V}$ has a finite subcover.

(b) There is an open cover $\mathscr{V}$ of $X,|\mathscr{V}|=\kappa$, such that for every open refinement $\mathscr{U}$ of $\mathscr{V}$, there is a point in $\kappa$ many $U \in \mathscr{U}$.

(c) $\kappa$ is the least ordinal such that $X \times(\kappa+1)$ is not normal.

Proof. (a) If $\mathscr{V}$ has no finite subcover, then $\{X \backslash V: V \in \mathscr{V}\}$ is a free closed filterbase.

(b) Let $\left\{H_{\alpha}: \alpha<\kappa\right\}$ and $\left\{p_{\zeta}: \zeta<\kappa\right\}$ be as in Proposition 2.3. Set $\Lambda=\{\lambda \in \kappa: \operatorname{cf}(\lambda)=\omega\}$. For $\lambda \in \Lambda$, define $M_{\lambda}=\bigcap\left\{H_{\alpha}: \alpha<\lambda\right\}$. Because $X$ is countably compact, for each $\lambda \in \Lambda$ we can find $q_{\lambda} \in M_{\lambda}$ such that for every neighborhood $U$ of $q_{\lambda},\left\{\alpha<\lambda: p_{\alpha} \in U\right\}$ is cofinal in $\lambda$. Now $\left\{X \backslash M_{\lambda}: \lambda \in \Lambda\right\}$ is an open cover of $X$; let $\mathscr{U}$ be an open refinement. Let $q_{\lambda} \in U_{\lambda} \in \mathscr{U}$. By the pressing down lemma, there is $p_{\alpha}$, which is in $U_{\lambda}$ for cofinally many $\lambda$ 's. Because $q_{\lambda} \notin M_{\lambda}$, there are $\kappa$ many different $U_{\lambda}$ 's.

(c) Follows from (a), (b), and Kunen's Theorem [P, 3.7]. We remark that the disjoint closed subsets of $X \times(\kappa+1)$ that cannot be separated are $X \times\{\kappa\}$ and $\bigcup\left\{M_{\lambda} \times\{\lambda\}: \lambda \in \Lambda\right\}$. 
One property of ordinals of uncountable cofinality is that if the "corner" is removed from the product of the compactifications of two such spaces, then the resulting space is nonnormal. The same thing holds for NAC spaces. This fact is a consequence of the following proposition, which we doubt is new but whose proof we have not seen in print.

Proposition 4.2. Suppose that $X$ and $Y$ are Tychonov spaces, $p \in \beta X \backslash X$, and $q \in \beta Y \backslash Y$. Then $(\beta X \times \beta Y) \backslash\{(p, q)\}$ is nonnormal.

Proof. We may assume without loss of generality that $X$ and $Y$ are almost compact. Otherwise, replace $X$ by $\beta X \backslash\{p\}$ and replace $Y$ by $\beta Y \backslash\{q\}$. The product of two locally compact, pseudocompact space is pseudocompact (see [W]) so by Glicksberg's product theorem, $\beta(X \times Y)=\beta X \times \beta Y$. Therefore, since $(\beta X \times \beta Y) \backslash\{(p, q)\}$ lies between a space and its Stone-Čech compactification and contains all but one point of the Stone-Čech remainder, $(\beta X \times \beta Y) \backslash\{(p, q)\}$ is almost compact. If it were also normal, it would be a NAC space and could not have two disjoint noncompact closed subsets; but it does have two such subsets: $X \times\{q\}$ and $\{p\} \times Y$.

According to Nogura's Theorem (see [P. 3.19]) if $X$ is an ordinal of uncountable cofinality $\kappa$ and $C$ is a compact space of tightness less than $\kappa$, then $X \times C$ is normal. This result cannot be proved (at least in ZFC) for arbitrary NAC spaces because by Tamano's theorem (see [P, 3.9]) $\Theta \times \beta \Theta$ is not normal even though $\tau(\beta \Theta)=\omega<\omega_{1}=\operatorname{cf}(\Theta)$. However, for spaces in $\mathrm{FR}(\kappa)$, the Nogura result does generalize. We first note that although Theorem 3.19 of [P] is stated in the special case that $X=\kappa^{+}$, the proof gives the following.

Proposition 4.3. Suppose $X \in \Omega(\kappa)$ and $C$ is a compact space such that $\tau^{+}(C)$ $\leq \kappa$. Then $X \times C$ is normal.

Proposition 4.4. Suppose that $X$ is a NAC space, $L$ is a closed, noncompact subset of $X$, and $C$ is compact. Then $X \times C$ is normal if and only if $L \times C$ is normal.

Proof. Sufficiency is clear.

For the other direction, we make the identifications $\beta L=L \cup\{\infty\} \subseteq X \cup$ $\{\infty\}=\beta X, \beta(L \times C)=\beta L \times C$, and $\beta(X \times C)=\beta X \times C$. Let $H_{e}, e \in 2$, be disjoint closed subsets of $X \times C$. For the rest of this paragraph, we fix $y \in C$ and omit subscripts $y$ on $e, U, V$, and $K$. Because $L \times C$ is normal, there are $e \in 2, U$ open in $X$, and $V$ open in $Y$ such that $(\infty, y) \in U \times V$ and $(\mathrm{Cl}(U \times V)) \cap(L \times C) \cap H_{e}=\varnothing$. Consider $K=\mathrm{Cl}\left(H_{e} \cap(U \times V)\right) \subseteq$ $H_{e} \cap \mathrm{Cl}(U \times V) \subseteq(X \backslash L) \times C$. Because the projection map $\pi_{X}$ is perfect, $\pi_{X}(K)$ is closed. Because $X$ is NAC and $\pi_{X}(K)$ is disjoint from $L, U \backslash \pi_{X}(K)$ is a neighborhood of $\infty$.

Let $\left\{V_{y}: y \in d\right\}$ be a finite subcover of $\left\{V_{y}: y \in C\right\}$. Note that $F=$ $\bigcup\left\{\left(X \times U_{y}\right) \cup \pi_{X}\left(K_{y}\right): y \in d\right\} \times C$ is compact, and that the sets $\pi_{C}\left(H_{e} \backslash F\right)$, $e \in 2$ have disjoint closures in $C$. Now it is routine cutting and pasting to separate $H_{0}$ and $H_{1}$. 
Theorem 4.5. Suppose that $X \in \mathrm{FR}(\kappa)$ and $C$ is compact. Then $X \times C$ is normal if and only if $\tau^{+}(C) \leq \kappa$.

Proof. Necessity follows from Theorem 4.1 and Katuta's Theorem [P, 3.16]. Sufficiency follows from Propositions 4.3 and 4.4.

The following results are a bit technical, so we begin with some background. A space $X$ is $A C R I N$ if all continuous regular images of $X$ are normal. Since every continuous image of a NAC space is either compact or NAC, every NAC space is ACRIN. In [FL2] it is proved that if $X$ is normal and $\beta X \backslash X$ is $C$ closed (that is, if every countably compact subset is closed), then $X$ is ACRIN. It was conjectured there that $C$-closed can be replaced by countable tightness. To find a counterexample, we must (1) find a space $S$ that has countabie tightness and is not $C$-closed; (2) find a normal space $X$ such that $\beta X \backslash X$ is homeomorphic to $S$; and (3) show that $X$ is not ACRIN. The only immediate candidates for $S$ are $\beta \Theta$ and an example of Fedorčuk, both of which require an extra axiom to construct, Indeed, PFA implies that locally compact spaces of countable tightness are sequential, hence $C$-closed [BDFN]. Above, we showed that $\omega_{1} \times \beta \Theta$ and $\delta(N, \kappa) \times \beta \Theta$ are normal. Below, we show that they are ACRIN, so the conjecture remains open.

Theorem 4.6. (a) Suppose that $Z$ is a normal $<\kappa$-bounded space such that $\tau^{+}(\beta Z \backslash Z) \leq \kappa$. Then $Z$ is $A C R I N$. In particular, if $L \in \Omega(\kappa), C$ is compact, and $\tau^{+}(C) \leq \kappa$, then $L \times C$ is $A C R I N$.

(b) If $X \in \mathrm{FR}(\kappa), C$ is compact, and $\tau^{+}(C) \leq \kappa$, then $X \times C$ is $A C R I N$. Proof. Let $f: Z \rightarrow Y$ be a continuous surjection. For the sake of clarity, we assume that $Y$ is Tychonov. The modifications needed in case $Y$ is merely regular are discussed in [FL2]. Let $\beta f: \beta Z \rightarrow \beta Y$ be the Stone extension of $f$. Let $P=\{p \in \beta Z \backslash Z: \beta f(p) \in Y\}$. By an argument in [FL2], it suffices to show that $P$ is a closed subset of $\beta Z \backslash Z$. Let $q$ be an element of $\beta Z \backslash Z$ in the closure of $P$. There is $A \in[P]^{<\kappa}$ such that $q \in \mathrm{Cl}_{\beta Z} A$. For $a \in A$, choose $z(a) \in Z$ so that $f(z(a))=\beta f(a)$. Now $K=\mathrm{Cl}_{Z}\{z(a): a \in A\}$ is compact, and by continuity, $\beta f(q) \in f^{\rightarrow}(K)$. Hence, $q \in P$ and $P$ is closed.

For part (b), let $Z=X \times C$ and define $\beta f, P$, and $q$ as above. Let $L \in \Omega(\kappa)$ be closed in $X$. Set $P^{\prime}=\left\{p \in \beta Z \backslash Z: \beta f(p) \in f^{\rightarrow}(L \times C)\right\}$. If $q \in \mathrm{Cl}_{\beta Z} P^{\prime}$, proceed as above choosing $A \in\left[P^{\prime}\right]^{<\kappa}$ and $z(a) \in L \times C$. If not, there is a closed neighborhood (in $\beta Z \backslash Z) H$ of $q$ such that $\beta f^{\rightarrow}(H) \cap$ $f^{\vec{\prime}}(L \times C)=\varnothing$. Let $K$ be the closed set $f^{\leftarrow}\left(\beta f^{\rightarrow}(H)\right)$. Note that $\pi_{X}(K)$ is closed and disjoint from $L$; hence, $\pi_{X}(K)$ and $K$ are compact. As above, $\beta f(q) \in f^{\rightarrow}(K)$. Therefore, $q \in P$ and $P$ is closed.

We can extend two results of [FL1] from ordinals of uncountable cofinality to NAC spaces. The proofs are essentially in [FL1].

Proposition 4.7. Suppose that $X$ is a NAC space.

(a) If $\mathrm{zf}(X) \mid \leq \mathbf{c}$, then the discrete union of $X$ and $\omega$ is not ACRIN.

(b) If $\operatorname{cf}(X)>\mathrm{c}$, then the discrete of $X$ and $\omega$ is $A C R I N$. 


\section{OTHER RESULTS}

In this section we talk about other results relating to NAC spaces. Discussions with Amer Bešlagić were very helpful, especially in this section. The cardinal $\mathbf{p}$ is defined in [vD2].

Proposition 5.1. Suppose that $X$ is a separable NAC space. Then $\mathbf{p} \leq \operatorname{zf}(X) \leq \mathbf{c}$. In particular, assuming $M A, \operatorname{zf}(X)=\mathbf{c}$.

Proof. Suppose $\left\{B_{\alpha}: \alpha<\lambda\right\}$ were a base at $\infty$, where $\lambda<\mathbf{p}$. Let $C=\left\{c_{k}\right.$ : $k \in \omega\}$ be a countable dense subset of $X$. Since $\lambda<\mathbf{p}$, there is an infinite subset $A$ of $C$ that is almost contained in every set of the form $C \cap B_{\alpha}$. Since $X$ is NAC, we are done if we can show that $A$ is a closed discrete subset of $X$. Suppose $z \in X$. Let $U$ and $B_{\alpha}$ be disjoint neighborhoods in $\beta X$ of $z$ and $\infty$ respectively. Since $A$ is almost contained in $B_{\alpha}$, only finitely many elements of $A$ are in $U$.

In view of Proposition 5.1, the following question seems natural. Does ZFC imply the existence of a separable NAC space $X$ with $\operatorname{cf}(X)=\mathrm{c}$ ?

Proposition 5.2. Assuming PFA, if $X \in \mathrm{NAC}\left(\omega_{1}\right)$, then in the construction of 2.3 , the points $p_{\zeta}$ can be chosen so that for $\alpha<\omega_{1}, \mathrm{Cl}_{X}\left\{p_{\zeta}: \zeta<\alpha\right\}$ is compact. Hence, $\operatorname{zf}(X)=\omega_{1}=\operatorname{cf}(X)$.

Proof. The result follows from the proof (but not the statement) of [BFDN]. See also [T, 8.14].

Now suppose that $X$ is an arbitrary Tychonov space and $p \in \beta X \backslash X$. If we view $p$ as a $z$-ultrafilter, it is possible that $p$ extends to a unique closed ultrafilter $p^{\sharp}$. In this case we can define $\operatorname{cf}_{p}(X)$, the cofinality at $p$, to be the minimal cardinality of a free filterbase included in $p^{\sharp}$, and $\mathrm{zf}_{p}(X)$ to be the least cardinality of a free filterbase included in $p$. Clearly, $\operatorname{cf}_{p}(X) \leq \mathrm{zf}_{p}(X)$.

Example 5.3. Assuming \&, de Caux showed in [dC] that there exist a Dowker space $X$ with the following properties.

(i) the underlying set is $\omega_{1} \times \omega$.

(ii) For every $\alpha \in \omega_{1}$ and $n \in \omega$, the set $\alpha \times n$ is open in $X$.

(iii) For every two uncountable subsets $A$ and $B$ of $X$ and $\alpha \in \omega_{1}, n \in \omega$, there are $\beta>\alpha$ and $m>n$ with $(\beta, m) \in \mathrm{Cl}_{X} A \cap \mathrm{Cl}_{X} B$.

Let $\mathscr{F}=\{A \subseteq X: A$ is closed and uncountable $\}$. Then (ii) and (iii) imply that $\mathscr{F}$ is a closed ultrafilter. Let $p=\{F \in \mathscr{F}: F$ is a zero set of $X\}$. by (ii), the sets $F_{n}=\omega_{1} \times(\omega \backslash n), n<\omega$, are in $\mathscr{F}$, so that $\operatorname{cf}_{p}(X)=\omega$ (since $\cap F_{n}=\varnothing$ ). By (iii) each open set containing an uncountable closed set has countable complement in $X$. Hence, each $F \in p$, being a $G_{\delta}$-set, has countable complement in $X$, so $\mathrm{zf}_{p}(X)>\omega$. Therefore, it is not true in ZFC that $\mathrm{zf}_{p}(X)=\mathrm{cf}_{p}(X)$.

On the other hand, certain results about NAC spaces do generalize to the "local" setting. The following can be proved using the methods of $\S 2$. 
Proposition 5.4. Suppose that $X$ is normal and locally compact and $p \in \beta X \backslash X$. Then $\operatorname{cf}_{p}(X) \leq \mathrm{zf}_{p}(X) \leq 2^{\mathrm{cf}_{p}}(X)$.

Example 5.5. Let $X$ be Rudin's ZFC Dowker space. Let $p: N \rightarrow \omega_{\omega}$ be defined by $p(n)=\omega_{n}$. Note [R, p. 185] that $p \in X^{\prime} \approx v X \subseteq \beta X$. It is easy to verify that $\operatorname{cf}_{p}(X)=\omega$ and $\mathrm{zf}_{p}(X)=\omega_{\omega}$, which is singular.

\section{REFERENCES}

[BDFN] Z. Balogh, A. Dow, D. Fremlin, and P. Nyikos, Countable tightness and proper forcing, Bull. Amer. Math. Soc. 19 (1988), 295-298.

[dC] P. de Caux, A collectionwise normal, weakly $\theta$-refinable Dowker space which is neither irreducible nor realcompact, Topology Proc. 1 (1976), 67-77.

[vD1] E. K. van Douwen, $A$ basically disconnected normal space $\Phi$ such that $|\beta \Phi \backslash \Phi|=1$, Canad. J. Math. 31 (1979), 911-914.

[vD2] _ The integers and topology, Handbook of Set-theoretic Topology (K. Kunen and J. Vaughan, eds.), North-Holland, Amsterdam, 1984, pp. 111-167.

[E] R. Engelking, General topology, Heldermann Verlag, Berlin, 1989.

[FL1] W. Fleissner and R. Levy, Ordered spaces all of whose continuous images are normal, Proc. Amer. Math. Soc. 105 (1989), 231-235.

[FL2] _ Stone-Čech remainders that make continuous images normal, Proc. Amer. Math. Soc. 106 (1989), 839-842.

[F] D. Fremlin, Perfect preimages of $\omega_{1}$ and the PFA, Topology Appl. 29 (1988), 151-166.

[FR] S. Franklin and M. Rajagopalan, Some examples in topology, Trans. Amer. Math. Soc. 155 (1971), 305-314.

[O] A. Ostaszewski, On countably compact, perfectly normal spaces, J. London Math. Soc. (2) 14 (1976), 505-516.

[P] T. Przymusinski, Products of normal spaces, Handbook of Set-theoretic Topology (K. Kunen and J. Vaughan, eds.), North-Holland, Amsterdam, 1984, pp. 781-826.

[R] M. E. Rudin, A normal space $X$ for which $X \times I$ is not normal, Fund. Math. 73 (1971), 179-186.

[T] S. Todorčević, Partition problems in topology, Contemp. Math., vol. 84, 1989.

[W] R. Walker, The Stone-Cech compactification, Springer-Verlag, New York, 1974.

Department of Mathematics, University of Kansas, LaWrence, KANSAS 66045

(J. Kulesza, R. Levy) Department of Mathematics, George Mason University, Fairfax, VIRGINIA 22030 